\section{Fatores de risco comportamentais para doenças cardiovasculares entre adolescentes da zona rural de um município do Sul do Brasil}

\author{
Behavioral risk factors for cardiovascular \\ diseases in adolescents from the rural area \\ of a municipality in Southern Brazil
}

\author{
Factores de riesgo comportamentales \\ para enfermedades cardiovasculares entre \\ adolescentes de la zona rural de un municipio \\ del sur de Brasil
}

Fernanda Borba dos Santos 1

Bruna Celestino Schneider 2

Marina Soares Valença 1

Nathalia Brandão Peter 1,3

Ludmila Correa Muniz 1 doi: 10.1590/0102-311X00241119

\section{Resumo}

O estudo objetivou estimar a prevalência de fatores de risco comportamentais para doenças cardiovasculares (DCV) e fatores associados ao seu acúmulo entre adolescentes da zona rural do Município de Pelotas, Rio Grande do Sul, Brasil. Estudo transversal, do tipo censo de base escolar, com amostra de 515 estudantes matriculados do 60 ao 9o ano do Ensino Fundamental das 21 escolas municipais rurais de Pelotas. Os fatores de risco comportamentais investigados foram: inatividade física; consumo irregular de frutas, legumes e verduras; experimentação de álcool e tabaco; e adição de sal à comida. $O$ desfecho do estudo foi o escore de aglomeração de fatores de risco comportamentais variando de 0 a 3: nenhum fator de risco comportamental para DCV ou exposição a 1,2 ou $\geq 3$ fatores de risco comportamentais. Realizou-se regressão logística ordinal para avaliar o efeito ajustado das características individuais sobre o acúmulo de fatores de risco comportamentais, tendo como categoria de referência os indivíduos sem qualquer fator de risco. A inatividade física foi o fator de risco mais prevalente (74,8\%), seguido do consumo irregular de frutas, legumes e verduras (56,2\%). Aproximadamente $42 \%$ dos adolescentes apresentaram dois fatores de risco comportamentais, sendo a combinação inatividade física e consumo irregular de frutas, legumes e verduras a mais prevalente (23\%). As chances de acumular dois ou mais fatores de risco comportamentais aumentou com a idade. A presença de fatores de risco comportamentais, bem como a simultaneidade destes é bastante elevada entre os adolescentes da zona rural do Município de Pelotas, evidenciando que eles estão expostos ao desenvolvimento de DCV. São necessárias intervenções preventivas de ocorrência simultânea desses fatores.

Adolescente; Doenças Cardiovasculares; Fatores de Risco; Zona Rural

\author{
Correspondência \\ N. B. Peter \\ Programa de Pós-graduação em Nutrição e Alimentos, \\ Universidade Federal de Pelotas. \\ Rua Gomes Carneiro 1, Pelotas, RS 96010-610, Brasil. \\ nathaliabpeter@gmail.com

\footnotetext{
1 Programa de Pós-graduação em Nutrição e Alimentos, Universidade Federal de Pelotas, Pelotas, Brasil.

2 Programa de Pós-graduação em Epidemiologia, Universidade Federal de Pelotas, Pelotas, Brasil.

3 Faculdade Anhanguera de Pelotas, Pelotas, Brasil.
} 


\section{Introdução}

As doenças crônicas não transmissíveis (DCNT) se desenvolvem no decorrer da vida como consequência de um conjunto de fatores. Tabagismo, atividade física insuficiente, alimentação não saudável e o uso nocivo de álcool estão entre os principais fatores de risco modificáveis para a maioria das DCNT 1. Dentre as principais DCNT estão as cardiovasculares (DCV) que, atualmente, configuram a maior causa de morte no mundo, principalmente em países de baixa e média renda 2. Segundo a Organização Mundial da Saúde (OMS), nos países em desenvolvimento, como o Brasil, as DCV foram responsáveis por mais de três quartos dos óbitos ocorridos nos últimos anos 2. A exposição precoce a determinados fatores de risco está associada ao desenvolvimento da maioria das DCNT, incluindo as DCV 3. Embora as manifestações clínicas da doença sejam observadas com maior frequência na idade adulta, a exposição aos fatores de risco para DCV vêm ocorrendo cada vez mais cedo, atingindo crianças e adolescentes 4 .

A etiologia da DCV é multifatorial, podendo decorrer de características biológicas do indivíduo e das condições socioeconômicas, ambientais, culturais e de urbanização do meio em que ele está inserido ${ }^{5}$. Além disso, diversos comportamentos, considerados fatores de risco comportamentais para a saúde cardiovascular, têm sido associados ao desenvolvimento das DCV 6. Dentre esses fatores destacam-se os hábitos alimentares não saudáveis, a inatividade física, o uso de tabaco e o consumo de álcool 2.

No Brasil, não existem trabalhos que evidenciem o acúmulo de fatores de risco comportamentais para DCV entre adolescentes de zona rural, principalmente na Região Sul do país, portanto, ainda não há conhecimento sobre o quanto estes jovens estão expostos ao desenvolvimento de DCV. No entanto, estudos nacionais realizados na zona urbana têm mostrado alta prevalência de fatores de risco comportamentais para DCV entre adolescentes, sendo a inatividade física e o consumo inadequado de frutas, legumes e verduras os comportamentos de risco mais prevalentes 7,8,9, referidos por cerca de $60 \% 10$ e $80 \% 11$ dos jovens, respectivamente. Outros comportamentos de risco, como o uso de álcool e tabaco também têm sido frequentemente observados nesse grupo etário 12 , alcançando a marca de $21 \%$ de relato de experimentação de álcool 13 e $18,5 \%$ de experimentação de tabaco 14 . Tal cenário é preocupante, visto que muitos desses comportamentos são mantidos após a adolescência tendo impacto negativo sobre a saúde dos indivíduos na vida adulta 12 .

O conhecimento da prevalência de cada fator isolado fornece apenas uma visão parcial do problema, considerando-se que, frequentemente, os fatores de risco comportamentais ocorrem de forma simultânea e que a presença concomitante destes comportamentos pode ser ainda mais prejudicial à saúde 15 . Um estudo transversal realizado com adolescentes identificou que cerca de $60 \%$ apresentaram um ou dois fatores de risco comportamentais simultaneamente, sendo o acúmulo mais prevalente em meninas, adolescentes mais velhos e de maior classe econômica 7.

Dessa maneira, a redução do risco para DCV, bem como a sua prevenção, ocorre por meio do controle de fatores comportamentais $2 \mathrm{e}$, para isto, identificar e compreender a distribuição destes fatores é fundamental 16,17 . Além disso, a maioria dos trabalhos que avaliam a ocorrência de fatores de risco comportamentais foram conduzidos com adolescentes residentes em áreas urbanas, sendo escassos os realizados na zona rural. E é sabido que cada vez mais os jovens da zona rural estão incorporando hábitos e comportamentos semelhantes aos dos jovens da área urbana 18,19. Diante disso, o objetivo do presente estudo é estimar a prevalência de fatores de risco comportamentais e fatores associados ao seu acúmulo entre adolescentes da zona rural do Município de Pelotas, Rio Grande do Sul, Brasil.

\section{Metodologia}

Trata-se de um estudo transversal, do tipo censo de base escolar, realizado com a comunidade escolar rural (estudantes do Ensino Fundamental, pais/responsáveis, professores, trabalhadores da alimentação escolar e gestores) do Município de Pelotas. À época do estudo, a zona rural do município contava com 21 escolas municipais, as quais ofereciam Ensino Fundamental para 2.326 alunos, sendo 922 matriculados do 6o ao 9o ano (dados fornecidos pela Secretaria Municipal de Educação e Desporto de 
Pelotas). Foram considerados elegíveis para o presente estudo todos os escolares matriculados do $6 \underline{0}$ ao 9o ano do Ensino Fundamental, das 21 escolas municipais rurais de Pelotas.

Cinco fatores de risco comportamentais para DCV foram operacionalmente definidos como:

(1) Inatividade física: foi considerada inatividade física a prática de menos de 300 minutos de atividade física no lazer na semana anterior à entrevista, segundo recomendações atuais 20. O tempo de atividade física no lazer foi mensurado por meio de uma lista de atividades físicas (futebol de sete, rua ou campo; futebol de salão (futsal); atletismo; basquete; jazz, balé, outras danças; vôlei; handebol; caçador; jogo de taco; outro esporte) questionadas ao adolescente sobre a prática (não; sim), dias na última semana $(1 ; 2 ; 3 ; 4 ; 5 ; 6 ; 7)$ e tempo (referido pelo estudante em horas e minutos).

(2) Experimentação de álcool: obtida pela pergunta: "Alguma vez na vida você experimentou bebida alcoólica? (não; sim)" 21. Aqueles que responderam "sim” foram considerados positivos para o desfecho. (3) Experimentação de tabaco: obtida pela pergunta: "Alguma vez na vida você fumou, mesmo uma ou duas tragadas? (não; sim)" 21. Aqueles que responderam "sim" foram considerados positivos para o desfecho.

(4) Consumo irregular de frutas, legumes e verduras: obtido com base em três perguntas: "Nos últimos 7 dias, em quantos dias você comeu salada crua (alface, tomate, cenoura, pepino, repolho ou outros)?”; "Nos últimos 7 dias, em quantos dias você comeu legumes e verduras cozidos (couve, abóbora, chuchu, brócolis, espinafre ou outros)?”; "Nos últimos 7 dias, em quantos dias você comeu frutas frescas ou salada de frutas?”. Foi considerado irregular o consumo de frutas, legumes e verduras inferior a 5 dias na semana anterior à entrevista 22.

(5) Adição de sal à comida: obtido baseando-se na pergunta: "Sem contar a salada, você costuma adicionar sal na comida pronta no seu prato?" (não; sim). Aqueles que responderam "sim” foram considerados positivos para o desfecho. Foi considerado como "costume", ou seja, o que o participante fazia na maioria das vezes.

As variáveis independentes utilizadas foram: sexo (masculino, feminino), idade (11-13, 14-19 anos), cor da pele (branca, não branca) e escolaridade dos pais/responsáveis $(0-4,5-8, \geq 9$ anos completos de estudos).

A coleta de dados ocorreu no período de fevereiro de 2015 a abril de 2016 . As informações sociodemográficas e aquelas referentes aos cinco fatores de risco comportamentais foram obtidas por meio de questionário preenchido pelo próprio aluno, em sala de aula, e por seus pais/responsáveis, para quem o questionário era enviado para ser respondido no domicílio e posteriormente retornava à equipe de pesquisa pelo escolar. O questionário dos pais/responsáveis continha informações sociodemográficas (anos de estudos; tipo e situação de trabalho; número de pessoas que moravam no domicílio), de saúde e comportamentais (consumo alimentar, hábito de fumar, doenças etc.).

Os dados foram duplamente digitados no pacote estatístico EpiData (http://www.epidata.dk/), para checagem de inconsistências, e analisados no programa Stata (https://www.stata.com). Inicialmente, foi realizada análise descritiva para a caracterização da amostra e cálculo das prevalências e respectivos intervalos de 95\% de confiança (IC95\%) dos fatores de risco comportamentais isolados para a amostra geral e por sexo. Posteriormente, foi criado um escore de aglomeração para retratar a presença de qualquer combinação entre os fatores, o qual variou de 0 a $3(0=$ nenhuma exposição; $1=$ exposição a um fator; 2 = exposição a dois fatores; e 3 = exposição a três ou mais fatores). Para detectar as diferenças entre os fatores de risco comportamentais acumulados e as características sociodemográficas dos estudantes, utilizou-se o teste qui-quadrado de Pearson de heterogeneidade ou de tendência linear, se a variável fosse categórica nominal ou ordinal, respectivamente. Por último, para verificar o efeito das características individuais sobre o acúmulo de fatores de risco comportamentais foram calculadas razões de odds (RO) brutas e ajustadas por meio de regressão logística ordinal, tendo como categoria de referência os indivíduos sem qualquer fator de risco (escore $=0$ ). As variáveis usadas para o ajuste foram sexo, idade, cor da pele e escolaridade dos pais/responsáveis, todas inseridas no mesmo nível hierárquico no modelo. Considerou-se um nível de 5\% de significância em todas as análises.

O estudo foi aprovado pelo Comitê de Ética em Pesquisa da Faculdade de Medicina, Universidade Federal de Pelotas (protocolo 950.128/2015). Fizeram parte do trabalho apenas os escolares cujos pais/responsáveis concordaram com a sua participação, mediante a assinatura do Termo de Consentimento Livre e Esclarecido. 


\section{Resultados}

Dos 922 alunos matriculados do 6o ao 9o ano do Ensino Fundamental das 21 escolas municipais rurais de Pelotas, os pais/responsáveis de 540 concordaram em participar da pesquisa. Desses, 517 preencheram o questionário. Foram consideradas perdas os alunos que não foram encontrados após duas tentativas na escola, e recusas, aqueles escolares cujos pais/responsáveis não concordaram em participar do trabalho. Dois participantes foram excluídos por terem 20 anos de idade, totalizando 515 estudantes analisados no presente estudo. A maioria dos adolescentes era do sexo masculino (52\%), tinha entre 11 e 13 anos de idade (61\%) e referiu ser de cor da pele branca (72,5\%). Aproximadamente $45 \%$ dos responsáveis pelos adolescentes estudaram entre 5 e 8 anos (Tabela 1).

A Figura 1 apresenta a prevalência isolada dos fatores de risco comportamentais para DCV para o total dos adolescentes e estratificado por sexo. $\mathrm{O}$ fatores de risco comportamentais mais prevalente foi inatividade física $(74,8 \%$; IC95\%: 70,8-78,3), sendo mais frequente entre as meninas ( $p=0,002)$. O consumo irregular de frutas, legumes e verduras foi o segundo fatores de risco comportamentais de maior prevalência, visto que mais da metade dos adolescentes (56,2\%; IC95\%: 51,8-60,4) relatou este comportamento, sendo mais prevalente entre os meninos ( $\mathrm{p}=0,008)$. A experimentação de álcool foi observada em 44,1\% (IC95\%: 39,8-48,5) dos adolescentes, seguida pela adição de sal à comida (17\%) e experimentação de tabaco $(5,8 \%)$, não havendo diferenças estatisticamente significativas entre meninos e meninas.

Quanto ao acúmulo de fatores de risco comportamentais, a maioria dos estudantes apresentou dois fatores de risco comportamentais (41,7\%; IC95\%: 37,4-46,2), não havendo diferença significativa entre os sexos. A combinação de dois fatores de risco comportamentais mais frequentes foi inatividade física e consumo irregular de frutas, verduras e legumes (55,1\%), seguida da inatividade física e experimentação de álcool (19,5\%) - dados não apresentados em tabelas.

A idade foi a única variável independente associada à distribuição do escore (Tabela 2). Maior prevalência de acúmulo de dois fatores de risco comportamentais foi observada entre os adolescentes com 11-13 anos de idade (47\%). Entre aqueles de 14-19 anos foi observada maior prevalência de três ou mais fatores de risco comportamentais $(39,1 \%)$, quando comparados aos mais jovens $(21,5 \%)$.

A Tabela 3 apresenta as RO brutas e ajustadas do escore de acúmulo de fatores de risco comportamentais segundo as características sociodemográficas. Observou-se que os adolescentes com 14 a 19 anos de idade apresentaram chance quase duas vezes maior de acumular mais de um fator de risco comportamental em relação àqueles com 11-13 anos ( $\mathrm{RO}=1,98$; IC95\%: 1,31-2,97).

\section{Tabela 1}

Características sociodemográficas dos adolescentes da zona rural. Pelotas, Rio Grande do Sul, Brasil, 2016 ( $\mathrm{n}=515$ ).

\begin{tabular}{|c|c|c|}
\hline Variáveis & $\mathbf{n}$ & $\%$ \\
\hline \multicolumn{3}{|l|}{ Sexo } \\
\hline Masculino & 268 & 52,0 \\
\hline Feminino & 247 & 48,0 \\
\hline \multicolumn{3}{|l|}{ Idade (anos) } \\
\hline $11-13$ & 315 & 61,0 \\
\hline 14-19 & 200 & 39,0 \\
\hline \multicolumn{3}{|l|}{ Cor da pele } \\
\hline Branca & 372 & 72,5 \\
\hline Não branca & 141 & 27,5 \\
\hline \multicolumn{3}{|c|}{ Escolaridade do responsável (anos completos de estudos) } \\
\hline $0-4$ & 122 & 32,0 \\
\hline $5-8$ & 177 & 45,6 \\
\hline 9 ou mais & 81 & 22,3 \\
\hline
\end{tabular}

* O percentual máximo de informações ignoradas foi de $26,2 \%$ ( $n=135)$ para a variável escolaridade do responsável. 


\section{Figura 1}

Prevalência de fatores de risco comportamentais para doenças cardiovasculares em adolescentes da zona rural. Pelotas, Rio Grande do Sul, Brasil, $2016(n=515)$.

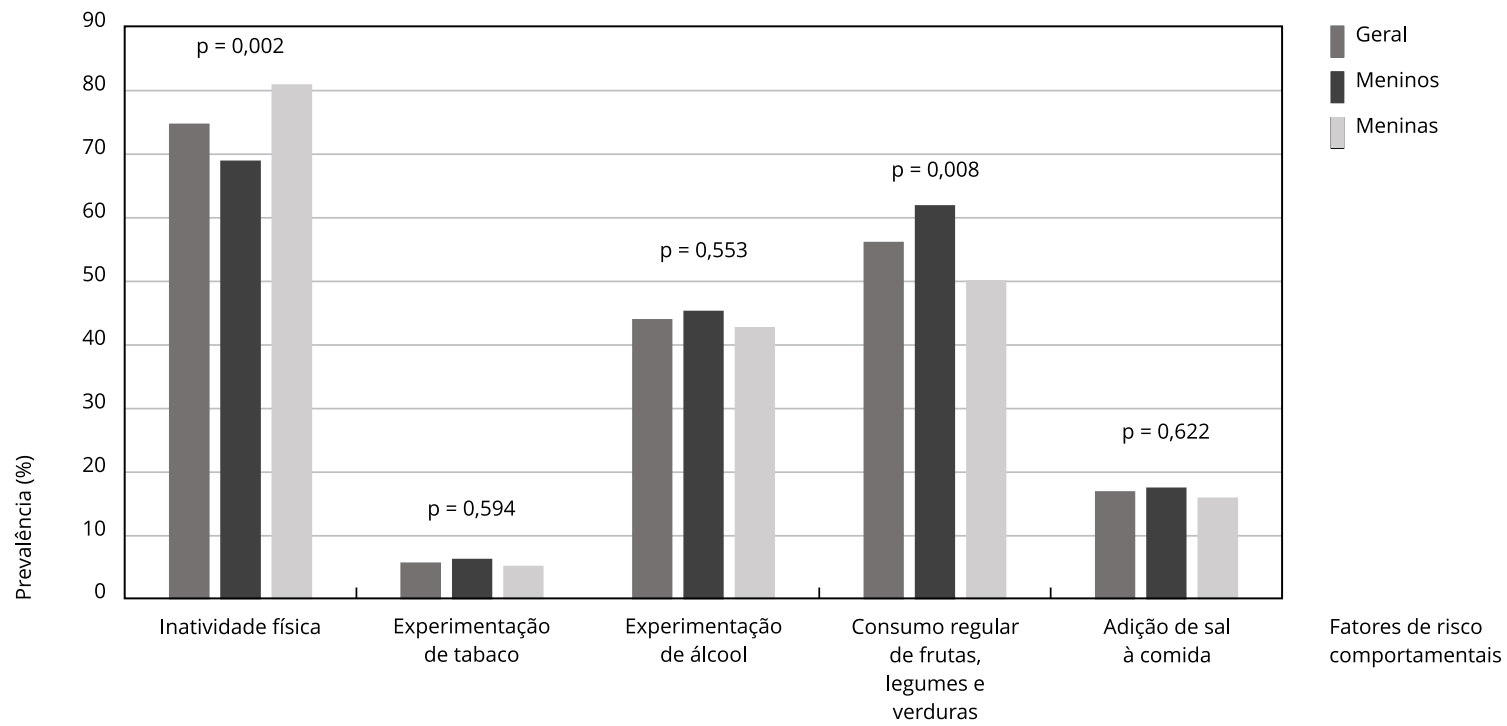

Nota: valores de $\mathrm{p}$ de acordo com o teste qui-quadrado de Pearson.

\section{Tabela 2}

Escore de acúmulo dos fatores de risco comportamentais para doenças cardiovasculares em adolescentes da zona rural, segundo variáveis sociodemográficas. Pelotas, Rio Grande do Sul, Brasil, 2016 ( $n=505)$

\begin{tabular}{|c|c|c|c|c|c|}
\hline \multirow[t]{2}{*}{ Variáveis } & \multicolumn{4}{|c|}{ Fatores de risco [\% (IC95\%)] } & \multirow[t]{2}{*}{ Valor de $p$} \\
\hline & 0 & 1 & 2 & $3 \mathrm{ou}+$ & \\
\hline Geral & $6,1(4,3-8,6)$ & $23,8(20,3-27,8)$ & $41,7(37,4-46,2)$ & $28,3(24,5-32,5)$ & \\
\hline Sexo & & & & & 0,368 * \\
\hline Masculino & $0,6(3,9-10,1)$ & $20,7(16,1-2,6)$ & $44,6(38,5-50,8)$ & $28,3(23,0-34,1)$ & \\
\hline Feminino & $5,8(3,4-9,6)$ & $27,0(21,8-33,0)$ & $38,8(32,7-45,0)$ & $28,3(22,9-34,3)$ & \\
\hline Idade (anos) & & & & & 0,000 * \\
\hline $11-13$ & $7,9(5,3-11,5)$ & $23,5(19,1-28,6)$ & $47,0(41,4-52,6)$ & $21,5(17,2-2,6)$ & \\
\hline $14-19$ & $3,2(1,4-6,8)$ & $24,3(18,7-30,9)$ & $33,3(26,9-40,3)$ & $39,1(32,4-46,3)$ & \\
\hline Cor da pele & & & & & 0,531 * \\
\hline Branca & $5,6(3,6-8,5)$ & $23,2(19,0-27,8)$ & $43,6(38,5-4,8)$ & $27,6(23,2-32,5)$ & \\
\hline Não branca & $7,6(4,1-13,6)$ & $25,2(18,4-33,3)$ & $36,6(28,8-45,2)$ & $30,5(2,3-38,9)$ & \\
\hline $\begin{array}{l}\text { Escolaridade do responsável (anos completos } \\
\text { de estudo) }\end{array}$ & & & & & $0,980 * *$ \\
\hline $0-4$ & $10,7(6,1-17,9)$ & $22,3(15,5-30,9)$ & $35,0(26,5-44,1)$ & $32,1(24,1-41,3)$ & \\
\hline $5-8$ & $6,5(3,6-11,3)$ & $21,3(15,7-28,1)$ & $44,4(37,0-52,0)$ & $27,8(21,6-35,1)$ & \\
\hline 9 ou mais & $2,5(0,6-9,6)$ & $29,1(20,1-40,0)$ & $41,7(31,4-52,9)$ & $26,6(17,9-37,4)$ & \\
\hline
\end{tabular}

IC95\%: intervalo de 95\% de confiança.

* Qui-quadrado de Pearson;

** Tendência linear. 
Tabela 3

Razão de odds (RO) bruta e ajustada do escore de acúmulo de fatores de risco comportamentais para doenças cardiovasculares em adolescentes da zona rural. Pelotas, Rio Grande do Sul, Brasil, 2016 ( $n=515)$.

\begin{tabular}{|c|c|c|c|c|}
\hline Variáveis & $\begin{array}{l}\text { RO bruta } \\
\text { (IC95\%) }\end{array}$ & Valor de $p$ * & $\begin{array}{c}\text { RO ajustada ** } \\
\text { (IC95\%) }\end{array}$ & Valor de $p$ * \\
\hline Sexo & & 0,460 & & 0,249 \\
\hline Feminino & Referência & & Referência & \\
\hline Masculino & $1,13(0,82-1,56)$ & & $1,25(0,85-1,84)$ & \\
\hline Idade (anos) & & 0,001 & & 0,001 \\
\hline $11-13$ & Referência & & Referência & \\
\hline $14-19$ & $1,76(1,25-2,48)$ & & $1,98(1,31-2,97)$ & \\
\hline Cor da pele & & 0,820 & & 0,192 \\
\hline Branca & $1,04(0,72-1,51)$ & & $1,34(0,86-2,07)$ & \\
\hline Não branca & Referência & & Referência & \\
\hline \multicolumn{5}{|c|}{ Escolaridade do responsável (anos completos de estudo) } \\
\hline $0-4$ & $1,01(0,60-1,72)$ & 0,958 & $0,92(0,54-1,58)$ & 0,773 \\
\hline $5-8$ & $1,07(0,67-1,73)$ & 0,780 & $1,03(0,63-1,67)$ & 0,909 \\
\hline 9 ou mais & Referência & & Referência & \\
\hline
\end{tabular}

IC95\%: intervalo de 95\% de confiança.

* Teste de Wald;

** Variáveis de ajuste: sexo, idade, cor da pele e escolaridade do responsável.

\section{Discussão}

Este trabalho identificou que mais de $90 \%$ dos adolescentes das escolas municipais da zona rural de Pelotas apresentaram pelo menos um fator de risco comportamental, sendo a inatividade física e o consumo irregular de frutas, legumes e verduras os fatores isolados mais prevalentes. Além disso, identificou-se que mais de $40 \%$ dos jovens acumularam pelo menos dois fatores de risco comportamentais e que a chance de acúmulo aumentou com a idade. No Brasil, há escassez de estudos que avaliam a prevalência de fatores de risco comportamentais em adolescentes residentes na zona rural, porém os resultados do presente trabalho são similares aos de estudos realizados na zona urbana $7,8,9,10$, evidenciando que apesar das diferenças sociodemográficas, econômicas, ambientais e comportamentais entre as duas áreas, o estilo de vida do meio rural está cada vez mais semelhante ao do urbano e que os adolescentes de zona rural estão expostos aos fatores de risco comportamentais para DCV tanto quanto os residentes de zona urbana.

Quando os fatores de risco comportamentais foram analisados de forma isolada, este estudo apontou a inatividade física como o fator mais prevalente entre os adolescentes da zona rural, principalmente entre as meninas. Pouco se conhece sobre a prática de atividade física no cenário rural, sobretudo entre adolescentes, visto que a maioria das evidências são provenientes de estudos conduzidos em zonas urbanas. Diante desses fatos, pode-se perceber a importância de desenvolver estudos cujo o objetivo seja investigar as barreiras, bem como os facilitadores para a prática de atividade física dos adolescentes da zona rural. No entanto, sempre existiu a hipótese de que a população rural é fisicamente ativa, devido à maior distância entre as casas, aos tipos de atividades de trabalho, que em sua maioria exige esforço físico, e as atividades de lazer, diferentes daquelas realizadas em área urbana 23. No mesmo sentido, em zona urbana, no interior do sul do Brasil, uma elevada prevalência de inatividade física foi observada entre adolescentes $(56,1 \%) 24$. As possíveis explicações para o aumento na prevalência de inatividade física podem ser atribuídas ao novo modo de vida das pessoas, que gastam mais tempo do lazer em frente às telas (TV, Internet, mídias sociais, videogames etc.) 25, juntamente com os aspectos sociais, como o aumento da violência 26 , e ambientais ou estruturais, como a ausência de quadras poliesportivas, espaços e equipamentos voltados à prática de esportes. 
O consumo irregular de frutas, legumes e verduras, frequente no presente trabalho, também é um problema verificado em outros estudos. A Pesquisa Nacional de Saúde do Escolar (PeNSE), conduzida com estudantes da rede pública das capitais brasileiras e Distrito Federal, mostrou que quase $70 \%$ dos alunos consumiam frutas de forma inadequada (consumo inferior a cinco dias na semana) 21. Tais evidências são preocupantes diante do fato de que os hábitos adquiridos na adolescência tendem a permanecer na vida adulta 27 , e que tanto a inatividade física quanto o consumo irregular de frutas, legumes e verduras estão entre os principais fatores de risco para a morbimortalidade em adultos 2 . Apesar dos adolescentes de zona rural estarem inseridos em um ambiente com maior acesso aos alimentos in natura, é nítida a mudança no consumo alimentar destes indivíduos, podendo ser justificada pela globalização que está cada vez mais presente em áreas rurais, responsável pela crescente oferta de alimentos industrializados, com preços mais acessíveis. Embora esses adolescentes tenham vasto acesso a alimentos mais saudáveis como frutas, legumes e verduras, pode-se deduzir que a carência de orientações sobre escolhas alimentares adequadas, por parte de profissionais da saúde em zonas rurais, também contribua para o baixo consumo destes alimentos. Portanto, a disseminação de orientações do Guia Alimentar para a População Brasileira 28 por parte dos profissionais de saúde em meio rural seria uma importante medida de estímulo à adoção de hábitos alimentares saudáveis, e consequente aumento do consumo de alimentos in natura como frutas, legumes e verduras.

A taxa de experimentação de álcool $(44,1 \%)$ e tabaco $(5,8 \%)$ no presente estudo foi semelhante à de outras pesquisas com adolescentes brasileiros 9,10. No Brasil, o uso de álcool entre adolescentes é elevado, e cerca de três vezes mais disseminado que o uso do tabaco 29. A PeNSE identificou prevalências de experimentação de álcool mais elevadas em capitais da Região Sul 30. Tais achados podem ser justificados por questões culturais, como a influência da colonização europeia e por ser uma região produtora cervejas e vinhos 13,21. A adolescência é uma fase da vida em que há intensa busca por aceitação e por novas experiências, que em conjunto com a massa de propagandas de bebidas alcoólicas que estimula a experimentação como um comportamento positivo, podem colaborar para as altas prevalências neste grupo etário 31 . Nas últimas décadas, pesquisas realizadas com escolares no Brasil apontaram que o tabaco ocupa o segundo lugar no ranking de drogas mais experimentadas no país, sendo a maior prevalência de experimentação entre os estudantes de escolas públicas 32 . De acordo com dados da PeNSE, a experimentação de tabaco entre os escolares de 13 a 15 anos de idade é de $19 \%$ 30. Neste trabalho, a prevalência de experimentação de tabaco entre os estudantes é a menor dentre os fatores estudados, apesar disto, ainda é preocupante que os adolescentes continuem expostos a este fator de risco comportamentais. No entanto, vale ressaltar o grandioso impacto que campanhas e ações antitabagismo desenvolvidas nas últimas décadas vêm exercendo sobre a população brasileira, em todas as faixas etárias 9 .

Quanto ao acúmulo de fatores de risco comportamentais, a elevada prevalência evidenciada neste estudo aponta para uma situação de risco à saúde dos adolescentes, pois a simultaneidade destes fatores expõe o indivíduo a um risco maior para DCV do que a presença de um fator isolado 33 . Adolescentes mais velhos foram identificados como grupo de risco para o maior acúmulo de fatores de risco comportamentais, assim como em um estudo transversal realizado com 1.628 estudantes de escolas públicas de Curitiba, Paraná, que mostrou que mais da metade dos jovens apresentavam um ou dois fatores de risco comportamentais para DCV simultaneamente, e que três em cada 10 adolescentes possuíam três ou mais fatores de risco comportamentais 7 , sendo a simultaneidade mais frequente entre os adolescentes mais velhos (de 13 a 17,9 anos). A adoção e o acúmulo de hábitos inadequados de saúde conforme o adolescente vai tornando-se mais velho podem ser justificados pela redução de limites sociais imposta pelos pais/responsáveis nesta fase. A crescente autonomia do adolescente nessa faixa etária aumenta a influência exercida pelas mídias e pelo meio em que está inserido. No entanto, é necessário que mais estudos investigando a relação entre essas duas variáveis sejam realizados, principalmente em grupos mais vulneráveis, como os jovens de zona rural.

Neste trabalho não houve associação entre o acúmulo de fatores de risco comportamentais para DCV e classe econômica medida pela escolaridade dos pais, divergindo de estudos conduzidos em zona urbana no Brasil e em outros países, que evidenciaram maiores chances de acumular fatores de risco comportamentais em adolescentes cujas famílias possuíam condições econômicas melhores 7,9,34,35. Cabe ressaltar que diferentes tipos de instrumentos são usados em estudos para estimar os níveis econômicos, podendo dificultar a comparação dos resultados. Além disso, a realidade 
da zona rural é diferente da urbana, podendo obter menores influências dos níveis econômicos sobre os fatores de risco comportamentais para DCV devido às especificidades no seu estilo de vida e ao custo de vida mais baixo.

O presente estudo tem como principal ponto forte a análise de fatores de risco comportamentais para DCV sob a perspectiva do ambiente escolar rural. Poucas publicações avaliaram as condições de saúde dessa população em âmbito nacional ou em municípios específicos, devido às dificuldades logísticas próprias destes locais e, no Município de Pelotas, estudos de censo escolar na zona rural sobre condições de saúde e nutrição são extremamente escassos.

Sabe-se que as populações rurais estão expostas a um grande conjunto de comportamentos de risco, o que torna-se ainda mais preocupante pelo fato de, muitas vezes, não possuírem tanto acesso à informação e aos serviços de saúde, como aqueles que vivem em áreas urbanas. Estudos brasileiros, realizados com adolescentes, mostram elevadas prevalências de fatores de risco comportamentais para DCV, porém, de forma isolada, poucos são aqueles que investigam a simultaneidade de fatores de risco comportamentais, principalmente em adolescentes de zona rural.

Por outro lado, as limitações devem ser discutidas. Inicialmente, a dificuldade logística influenciou diretamente no número de perdas e recusas, que apesar de elevado é inferior a outros estudos realizados no âmbito escolar 36,37,38. Além disso, vale ressaltar que as perdas e recusas referentes aos questionários dos pais/responsáveis dos adolescentes não foram calculadas devido à falta de informação relativa às famílias, uma vez que estes dados não eram disponibilizados pela Secretaria Municipal de Educação (SMED) ou pelas escolas.

Outra limitação é o fato de os fatores de risco comportamentais terem sido mensurados por autorrelato do adolescente, sendo comum a superestimação da prática de atividade física, bem como a subestimação do uso de álcool e tabaco. Ainda, a comparabilidade com outros estudos foi uma dificuldade em vista da falta de consenso entre as pesquisas quanto à definição de cada um dos fatores de risco comportamentais. Além disso, por ser um estudo com delineamento transversal é necessário cautela na interpretação dos resultados, pois, neste caso, uma relação de associação não sugere, necessariamente, uma relação de causalidade.

\section{Conclusão}

Diante das evidências deste estudo, conclui-se que os adolescentes da zona rural do Município de Pelotas apresentam altas prevalências de diversos fatores de risco comportamentais para o desenvolvimento de DCV, sendo a simultaneidade destes fatores mais frequente entre os mais velhos.

Sob a perspectiva de saúde pública esses dados mostram a importância de se aumentar a vigilância sobre cada um dos comportamentos avaliados em adolescentes rurais, além de incentivar os profissionais da saúde que têm acesso a este grupo a promover a prática de educação em saúde no intuito de estimular, dentro do contexto local, o consumo alimentar saudável e consciente, a prática regular de atividade física e desencorajar a experimentação de álcool e tabaco. Também é evidente a necessidade de mais estudos sobre o tema, uma vez que parece que os jovens da zona rural são tão expostos aos comportamentos de risco para DCV quanto àqueles que residem em zonas urbanas. Além disso, os dados dessas pesquisas irão subsidiar a elaboração de políticas públicas e programas de prevenção voltados à população rural jovem, uma vez que comportamentos de risco são passíveis de mudanças, e quanto mais precoce for a intervenção menor será o impacto negativo em fases futuras. 


\section{Colaboradores}

F. B. Santos, B. C. Schneider e L. C. Muniz contribuíram na concepção do projeto, análise e interpretação dos dados e redação do artigo. M. S. Valença e N. B. Peter contribuíram na revisão final do manuscrito e aprovação da versão para publicação.

\section{Informações adicionais}

ORCID: Fernanda Borba dos Santos (0000-00021393-9792); Bruna Celestino Schneider (00000002-1035-0106); Nathalia Brandão Peter (00000002-1769-4438); Marina Soares Valença (00000002-7830-5758); Ludmila Correa Muniz (00000002-4270-7704).

\section{Referências}

1. Ministério da Saúde. Sobre a vigilância de DCNT. http://portalms.saude.gov.br/noti cias/43036-sobre-a-vigilancia-de-dcnt (acessado em 28/Set/2018).

2. Organização Pan-Americana da Saúde; Organização Mundial da Saúde. Doenças cardiovasculares. https://www.paho.org/bra/ index.php?option $=$ com_content $\&$ view $=$ arti cle\&id=5253: doencas- cardiovasculares\&Ite mid=839 (acessado em 12/Jul/2018).

3. Fundo das Nações Unidas para a Infância. O estado das crianças do mundo. A adolescência: uma era de oportunidades. Brasília: Fundo das Nações Unidas para a Infância; 2011.

4. Freedman DS, Patel DA, Srinivasan SR, Chen $\mathrm{W}$, Tang R, Bond MG, et al. The contribution of childhood obesity to adult carotid intimamedia thickness: the Bogalusa Heart Study. Int J Obes (Lond) 2008; 32:749-56.

5. Pan American Health Organization. A network for integrated prevention \& control of chronic noncommunicable diseases (CNCDs) in the Americas. http://www.paho.org/eng lish/ad/dpc/nc/carmen-info.htm (acessado em 18/Jul/2018).

6. Di thiene D, Marceca M. Closing the gap in a generation: health equity through action on the social determinants of health. A challenge for the international community. Ann Ig 2008; 20:595-601.

7. Barbosa Filho VC, Campos W, Bozza R, Lopes AS. The prevalence and correlates of behavioral risk factors for cardiovascular health among Southern Brazil adolescents: a cross-sectional study. BMC Pediatr 2012; 12:130.

8. Romanzini M, Reichert FF, Lopes AS, Petroski EL, Farias Júnior JC. Prevalência de fatores de risco cardiovascular em adolescentes. Cad Saúde Pública 2008; 24:2573-81.

9. Farias Júnior FC, Mendes JKF, Barbosa DBM, Silva Lopes AS. Fatores de risco cardiovascular em adolescentes: prevalência e associação com fatores sociodemográficos. Rev Bras Epidemiol 2011; 14:50-62.

10. Costa IFAF, Medeiros CCM, Costa FDAF, Farias CRL, Souza DR, Adriano WS, et al. Adolescentes: comportamento e risco cardiovascular. J Vasc Bras 2017; 16:205-13.

11. Lancarotte I, Nobre MR, Zanetta R, Polydoro M. Estilo de vida e saúde cardiovascular em adolescentes escolares de São Paulo. Arq Bras Cardiol 2010; 95:61-9.

12. World Health Organization. Global health risks: mortality and burden of disease attributable to selected major risks. Geneva: World Health Organization; 2011.

13. Coutinho ESF, França-Santos D, Magliano ES, Bloch KV, Barufaldi LA, Cunha CF, et al. ERICA: padrões de consumo de bebidas alcoólicas em adolescentes brasileiros. Rev Saúde Pública 2016; 50 Suppl 1:8s. 
14. Figueiredo VC, Szklo AS, Costa LC, Kuschnir MCC, Silva TLN, Bloch KV, et al. ERICA: prevalência de tabagismo em adolescentes brasileiros. Rev Saúde Pública 2016; 50 Suppl 1:12s.

15. Khaw KT, Wareham N, Bingham S, Welch A, Luben R, Day N, et al. Combined impact of health behaviours and mortality in men and women: the EPIC-Norfolk prospective population study. PLoS Med 2008; 5:e70.

16. Quadros TMB, Gordia AP, Silva LR, Silva DAS, Mota J. Inquérito epidemiológico em escolares: determinantes e prevalência de fatores de risco cardiovascular. Cad Saúde Pública 2016; 32:e0181514.

17. Gidding SS. American Heart Association: dietary recommendations for children and adolescents: a guide for practitioners. Pediatrics 2006; 117:544-59.

18. Regis MF, Oliveira LMFT, Santos ARM, Leonidio ACR, Diniz PRB, Freitas CMSM. Estilos de vida urbano versus rural em adolescentes: associações entre meio-ambiente, níveis de atividade física e comportamento sedentário. Einstein (São Paulo) 2016; 14:461-7.

19. Troian A, Breitenbach R. Jovens e juventudes em estudos rurais do Brasil. Interações 2018; 19:789-802.

20. World Health Organization. Global recommendations on physical activity for health. Geneva: World Health Organization; 2010.

21. Malta DC, Oliveira-Campos M, Prado RR, Andrade SSC, Mello FCM, Dias AJR, et al. Tendência dos fatores de risco e proteção de doenças crônicas não transmissíveis em adolescentes, Pesquisa Nacional de Saúde do Escolar (PeNSE 2009 e 2012). Rev Bras Epidemiol 2014; 17:77-91.

22. World Health Organization. Diet, nutrition and the prevention of chronic diseases: report of a joint WHO/FAO expert consultation. Geneva: World Health Organization; 2003.

23. Martins RC, Silva ICM, Hallal PC. Atividade física na população rural de Pelotas - RS: prevalência e fatores associados. Rev Saúde Pública 2018; 52 Suppl 1:9s.

24. Cureau FV, Silva TLN, Bloch KV, Fujimori E, Belfort DR, Carvalho KMB, et al. ERICA: inatividade física no lazer em adolescentes brasileiros. Rev Saúde Pública 2016; 50 Suppl 1:4s.

25. Hallal PC, Andersen LB, Bull FC, Guthold R, Haskell W, Ekelund U. Global physical activity levels: surveillance progress, pitfalls and prospects. Lancet 2012; 380:247-57.

26. Scorzafave LG, Justus M, Shikida PFA. Safety in the global south: criminal victimization in Brazilian rural areas. J Rural Stud 2015; 39:247-61.

27. Azevedo MR, Araujo CL, Silva MC, Hallal PC. Tracking of physical activity from adolescence to adulthood: a population-based study. Rev Saúde Pública 2007; 41:69-75.
28. Departamento de Atenção Básica, Secretaria de Atenção à Saúde, Ministério da Saúde. Guia alimentar para a população brasileira. 2a Ed. Brasília: Ministério da Saúde; 2014.

29. World Health Organization. Social determinants of health and well-being among young people. Health Behaviour in School-aged Children (HBSC) study: international report from the 2009/2010 survey. Copenhagen: WHO Regional Office for Europe; 2012.

30. Instituto Brasileiro de Geografia e Estatística. Pesquisa Nacional de Saúde Escolar 2015. https://biblioteca.ibge.gov.br/visuali zacao/livros/liv97870.pdf (acessado em 08/ Mar/2018).

31. Vendrame A, Pinsky I, Faria R, Silva R. Apreciação de propagandas de cerveja por adolescentes: relações com a exposição prévia às mesmas e o consumo de álcool. Cad Saúde Pública 2009; 25:359-65.

32. Instituto Nacional de Câncer. Dados e números da prevalência do tabagismo. https://www. inca.gov.br/observatorio-da-politica-nacio nal-de-controle-do-tabaco/dados-e-nume ros-prevalencia-tabagismo (acessado em 21/ Mai/2020).

33. Odunaiya NA, Grimmer K, Louw QA. High prevalence and clustering of modifiable CVD risk factors among rural adolescents in southwest Nigeria: implication for grass root prevention. BMC Public Health 2015; 15:661.

34. Park YD, Patton LL, Kim HY. Clustering of oral and general health risk behaviors in Korean adolescents: a national representative sample. J Adoles Health 2010; 47:277-81.

35. Richter M, Vereecken CA, Boyce W, Ma L, Gabhainn SN, Currie CE. Parental occupation, family affluence and adolescent health behaviour in 28 countries. Int J Public Health 2009; 54:203-12.

36. Oliveira RR, Peter NB, Muniz LC. Consumo alimentar segundo grau de processamento entre adolescentes da zona rural de um município do sul do Brasil. Ciênc Saúde Colet 2019; http://www.cienciaesaudecoletiva.com.br/en/ articles/food-consumption-according-to-de gree-of-processing-among-adolescentsthe-ru ral-area-of-a-municipality-in-the-southof-brazil/17268.

37. Iepsen AM, Silva MC. Prevalência e fatores associados à insatisfação com a imagem corporal de adolescentes de escolas do Ensino Médio da zona rural da região sul do Rio Grande do Sul, 2012. Epidemiol Serv Saúde 2014; 23:317-25.

38. Müller WA, Silva MC. Barreiras à prática de atividades físicas de adolescentes escolares da zona rural do sul do Rio Grande do Sul. Rev Bras Ativ Fis Saúde 2013; 18:344-53. 
Abstract

The study aimed to estimate the prevalence of behavioral risk factors for cardiovascular diseases (CVDs) and factors associated with their accumulation in adolescents from the rural area of a municipality in Pelotas, Rio Grande do Sul State, Brazil. This was a cross-sectional school-based census-type study with a sample of 515 sixth to nineth-grade schoolchildren in 21 rural public schools in Pelotas. The target behavioral risk factors were: physical inactivity; irregular consumption of fruits, vegetables, and greens; initiation to alcohol consumption and smoking; and addition of salt to foods. The study outcome was the cluster score of behavioral risk factors, varying from 0 to 3: no behavioral risk factors for CVD or exposure to 1 , 2, or $\geq 3$ behavioral risk factors. Ordinal logistic regression was performed to assess the effect adjusted for individual characteristics on the accumulation of behavioral risk factors, using as the reference category individuals without any risk factor. Physical inactivity was the most prevalent risk factor (74.8\%), followed by irregular consumption of fruits, vegetables, and greens (56.2\%). Approximately 42\% of the adolescents presented two behavioral risk factors, the most prevalent combination of which was physical inactivity and irregular consumption of fruits, vegetables, and greens (23\%). The odds of accumulating two or more behavioral risk factors increased with age. The presence and simultaneity of behavioral risk factors is quite high among adolescents from the rural area of Pelotas, showing that they are exposed to the development of CVD. Interventions are needed to prevent the simultaneous occurrence of these risk factors.

Adolescent; Cardiovascular Diseases; Risk Factors; Rural Areas

\section{Resumen}

El objetivo del estudio fue estimar la prevalencia de factores de riesgo comportamentales para enfermedades cardiovasculares (DCV por sus siglas en portugués) y factores asociados a su acumulación entre adolescentes de la zona rural del Municipio de Pelotas, Rio Grande do Sul, Brasil. Estudio transversal, tipo censo de base escolar, con muestra de 515 escolares matriculados del 60 al $9 \underline{0}$ año de Enseñanza Fundamental de las 21 escuelas municipales rurales de Pelotas. Los factores de riesgo comportamentales investigados fueron: inactividad física; consumo irregular de frutas, legumbres y verduras; experimentación de alcohol y tabaco y adición de sal a la comida. El resultado del estudio fue la puntuación de aglomeración de factores de riesgo comportamentales, variando de O a 3: ningún factores de riesgo comportamentales para DCV o exposición a 1, $20 \geq 3$ factores de riesgo comportamentales. Se realizó regresión logística ordinal, para evaluar el efecto ajustado de las características individuales sobre el acúmulo de factores de riesgo comportamentales, teniendo como categoría de referencia los individuos sin cualquier factor de riesgo. La inactividad física fue el factor de riesgo más prevalente (74,8\%), seguido del consumo irregular de frutas, legumbres $y$ verduras (56,2\%). Aproximadamente un $42 \%$ de los adolescentes presentaron dos factores de riesgo comportamentales, siendo la combinación inactividad física y consumo irregular de frutas, legumbres y verduras la más prevalente (23\%). Las oportunidades de acumular dos o más factores de riesgo comportamentales aumentaron con la edad. La presencia de factores de riesgo comportamentales, así como la simultaneidad de estos es bastante elevada entre los adolescentes de la zona rural del Municipio de Pelotas, evidenciando que ellos están expuestos al desarrollo de DCV. Son necesarias intervenciones preventivas para la ocurrencia simultánea de estos factores.

Adolescente; Enfermedades Cardiovasculares;

Factores de Riesgo; Medio Rural
Recebido em 17/Dez/2019

Versão final reapresentada em 15/Jun/2020

Aprovado em 27/Jul/2020 